Research Paper

\title{
Upregulation of LINC01503 promotes cervical cancer progression by targeting the miR-615-3p/CCNDI axis
}

\author{
Jing Feng, Fang-yuan Gao, Yan-ying Li, Xi-yun Xiao, Jie Xi ${ }^{\bowtie}$ \\ Department of Gynecology, Cangzhou Central Hospital, Hebei, 061001, China. \\ $\triangle$ Corresponding author: Jie Xi, Department of Gynecology, Cangzhou Central Hospital, No.16 Xinhua Road, Hebei, China, 061001. E-mail: \\ dr_jiexi@yzpc.edu.cn or xijie512jiexi@163.com
}

(1) The author(s). This is an open access article distributed under the terms of the Creative Commons Attribution License (https://creativecommons.org/licenses/by/4.0/). See http://ivyspring.com/terms for full terms and conditions.

Received: 2020.10.06; Accepted: 2021.04.28; Published: 2021.06.01

\begin{abstract}
Mounting evidence indicates that long non-coding RNAs influence the progression of cervical cancer, but the precise function of LINC01503 in the pathogenesis of the disease remains unknown. Here, we found higher levels of LINC01503 in cervical cancer tissues. High LINC01503 expression was associated with enhanced progression of cervical cancer as indicated by advanced FIGO stage, increased metastasis of tumor cells to lymph nodes, and invasion into deeper cervical tissues. LINC01503 inhibition markedly suppressed the invasion and proliferative ability of tumor cells. Mechanistically, LINC01503 was demonstrated to negatively modulate the expression of miR-615-3p in cervical cancer. CCNDI was found to be a target of miR-615-3p. Rescue experiments indicated that LINC01503 inhibition suppressed the invasion and proliferative ability of the tumor cells, a phenomenon that was reversed following miR-615-3p inhibition or CCNDI overexpression. Collectively, these data indicate that LINC01503 enhances the progression of cervical cancer cells via interaction with miR-615-3p/CCNDI axis.
\end{abstract}

Key words: cervical cancer, LINC01503, miR-615-3p, CCND1

\section{Introduction}

Cervical cancer (CC) is the second most prevalent malignancy affecting women globally $[1,2]$. Technological advancements have greatly improved diagnosis and treatment of $\mathrm{CC}$, but the clinical outcomes remain poor as a result of metastasis/recurrence of the disease [3, 4]. Therefore, it necessary to elucidate the pathogenesis and molecular mechanisms of CC and develop strategies to improve treatment outcomes.

Long non-coding RNAs (lncRNAs) belongs to a class of non-coding RNAs consisting of $>200$ nucleotides $[5,6]$. Multiple reports show that lncRNAs regulates various cellular processes consisting of proliferation, apoptosis, cell cycle progression, as well as invasion. LncRNAs are thought to exert their influence via chromatin remodeling, transcriptional control, and post-transcriptional regulation [7-9]. Huang et al. reported that IncRNA MALAT1 can bind to the chromatin remodeling subunit BRG1 to epigenetically enhance the progression of inflammation-induced liver cancer [10]. Xiu et al. found that lncRNA TUG1 inhibition enhances radio-sensitivity of prostate cancer cells [11]. A study by $\mathrm{Hu}$ et al., showed that IncRNA ROR sponged miR-138 and regulated Mst1, thereby exacerbating apoptosis of cardiomyocytes triggered by hypoxia or reoxygenation [12]. The precise function of LINC01503 in the pathogenesis of $\mathrm{CC}$ remains unknown.

Here, we examined the role of LINC01503 in CC. Our findings confirm that this lncRNA facilitates the invasion as well as the proliferation of CC cells by interacting with the miR-615-3p/CCND1 axis.

\section{Materials and methods}

\subsection{Patients and specimens}

Human CC tissues and matched normal tissues were collected from 43 patients treated at Cangzhou Central Hospital and were confirmed by 2 
pathologists according to the guidelines of FIGO (International Federation of Gynecology and Obstetrics). None of the study subjects received any treatment (chemotherapy or radiotherapy) prior to surgery. All participants provided written informed consent. After collection, the specimens were briefly frozen in liquid nitrogen, and then preserved at $-80^{\circ} \mathrm{C}$. The ethics committee of Cangzhou Central Hospital approved the study. The clinical features of all the subjects are indicated in Table 1.

Table 1: The clinicopathological characteristics of cervical cancer patients.

\begin{tabular}{lll}
\hline Characteristics & & Number \\
\hline Age & $<50$ & 28 \\
& $\geq 50$ & 15 \\
Tumor size $(\mathrm{cm})$ & $<4$ & 26 \\
Depth of cervical invasion & $\geq 4$ & 17 \\
& $<2 / 3$ & 20 \\
Histological type & $\geq 2 / 3$ & 23 \\
Lymph node metastasis & Adenocarcinoma & 13 \\
& Squamous cell carcinoma & 30 \\
Figo stage & No & 24 \\
& Yes & 19 \\
& I & 9 \\
& II & 13 \\
& III & 10 \\
\hline
\end{tabular}

\subsection{Cell culture and transfection}

The CC cell lines consisting of $\mathrm{SiHa}, \mathrm{HeLa}, \mathrm{C} 33 \mathrm{~A}$, and CaSki, as well as the Ect1/E6E7 normal human cervical cell line were procured from ATCC. Subsequently, the cells were grown in RPMI 1640 medium (Gibco) enriched with 10\% fetal bovine serum (FBS) under a humidified environment with $5 \% \mathrm{CO}_{2}$ and $37^{\circ} \mathrm{C}$.

siRNA against LINC01503 (si-LINC01503\#1: 5'UCGGAAUACCCACCUUUCUGGUAAU-3';

si-LINC01503\#2: 5'- UGACAAGUGUGUACCUAC GUGUCAG-3'; si-LINC01503\#3: 5'- CAUGACCGU GUGGAGAAAGUUCUUU-3') and a non-targeting siRNA (si-NC: 5'-AATTCTCCGAACGTGTCACGT3') were supplied by GenePharma (Shanghai, China). RiboBio (Guangzhou, China) supplied the miR-615-3p mimics/inhibitor and matched controls. CCND1 expression plasmid was constructed in a pcDNA3.1 vector (Thermo Fisher Scientific, Waltham, MA, USA). Lipofectamine 2000 was employed for cell transfection as described by the manufacturer.

\subsection{Cell proliferation assessment}

The CCK-8 kit (Dojindo, Kumamoto, Japan) was employed to explore cell proliferation. In brief, transfected CC cells suspected in $500 \mu \mathrm{L}$ of media were cultured in 96-well plates for 24,48 , or 72 hours. $10 \mu \mathrm{L}$ of CCK8 solution was introduced into each well and cultured for further 2 hours. Subsequently, a microplate reader was employed to determine the absorbance at $450 \mathrm{~nm}$.

Colony formation assays were done by seeding 5000 transfected CC cells into 6-well plates. Subsequently, the cells were cultured for 14 days, followed by fixation with methanol for 30 minutes. Afterwards, a staining step was conducted using by $0.1 \%$ crystal violet before counting colony numbers.

\subsection{Transwell invasion assay}

Inoculation of the transfected cells suspended in serum-free medium into the upper chamber of the transwell membrane (BD Biosciences) was performed. The medium containing $10 \%$ FBS was put in the bottom chamber. 24 hours later, the cells were stained in the dark with crystal violet stain (Sigma, MO, USA), after which the cells were counted.

\subsection{Gene expression studies}

TRIzol reagent (Thermo Fisher Scientific) was employed to isolate RNA from the cells as per the protocols described by the manufacturer. The RNA was converted into cDNA with a cDNA Synthesis kit (Revert Aid First Strand, Fermentas, Canada). PCR was done using SYBR Premix Ex Taq II kit (Takara, Tokyo, Japan) on a CFX96 Real-Time PCR machine (Biorad) using the following program: $95^{\circ} \mathrm{C}$ for 6 minutes (denaturation); and 40 cycles of $94^{\circ} \mathrm{C}$ for 30 seconds (initiation), $60^{\circ} \mathrm{C}$ for 30 seconds (annealing), and $73^{\circ} \mathrm{C}$ for 1.5 minutes (elongation). Relative gene expression was determined via the $2^{-\Delta \Delta \mathrm{Ct}}$ approach. U6 along with GAPDH were served as the internal control.

\subsection{Western blot assay}

RIPA buffer (Sigma) enriched with 1mM PMSF was used to lyse the cells. A BCA assay kit (Thermo Fisher Scientific) was utilized to measure the protein concentration. Next, the proteins were resolved with SDS-PAGE, and then blotted onto PVDF membranes. After that, 5\% skimmed milk was utilized to prevent non-specific binding on the membranes for 1 hour, and overnight incubation was conducted at $4^{\circ} \mathrm{C}$ with anti-CCND1 primary antibody. The membranes were rinsed and incubated with HRP-labelled goat anti-rabbit secondary antibody (Abcam) and incubated at room temperature. The signal was then developed using enhanced chemiluminescence reagent (Advansta, MO, USA). Finally, the ImageJ was employed to quantify the proteins and the GAPDH gene utilized as the normalization control.

\subsection{Dual-luciferase reporter analysis}

Wildtype (WT) and mutant (MUT) LINC01503 or CCND1 3'UTR were amplified, then cloned into the pmirGlO luciferase reporter vector (Promega, 
Madison, WI, USA) to create WT-LINC01503, WT-CCND1, MUT-LINC01503, and MUT-CCND1 vectors. Cells were co-introduced into these reporters and miR-615-3p (or miR-NC) via co-transfection and cultured for 48 hours. Dual-luciferase assays (Promega) were then carried out to determine the luciferase activity and normalized to Renilla luciferase activity.

\subsection{RIP assay}

The Magna RNA binding protein immunoprecipitation kit (Millipore, Billerica, MA, USA) was utilized for the RIP assay. In summary, whole-cell lysates were prepared using RIP buffer, and then incubation with magnetic beads hybridized with human anti-Ago2 antibody or normal mouse IgG as a negative control performed. The sample was then incubated with proteinase $\mathrm{K}$, followed by RNA immunoprecipitation. The immunoprecipitated RNA was purified and analyzed by RT-qPCR to quantify LINC01503 and miR-615-3p expression.

\subsection{Nude mouse model in vivo}

The Animal Care and Use Committee of Cangzhou Central Hospital approved all the mice experiments. We subcutaneously administered $2 \times 10^{6}$ stably transfected cells into BALB/C 4-week-old female nude mice weighing $18-25 \mathrm{~g}(\mathrm{n}=4$ for each group). Tumor volume was monitored using a caliper weekly from the $7^{\text {th }}$ day after injection. Tumor volume was computed via the formula: length $\times$ width $^{2} \times 0.5$. The animals were euthanized six weeks after xenografting using cervical dislocation, the tumors weights were measured for downstream analyses.

\subsection{Bioinformatics analysis}

The present study determined LINC01503 expression into the GEPIA dataset to explore its differential expression and influence on the prognosis of individuals with CESE, OV, UCEC, and UCS. TargetScan, miRcode, LncBase v.2 and Starbase V2.0 were used to investigate the potential target of LINC01503. TargetScan, miRWalk as well as miRTarBase were employed in predicting the prospective target gene of miR-615-3p.

\subsection{Data analysis}

Data analysis was conducted with the SPSS version 20.0. All data are indicated as mean \pm standard deviation (SD). Student's t-test was employed to determine differences between 2 groups. One-way ANOVA followed by Tukey test (post-hoc) was utilized to analyze differences across multiple groups. $\mathrm{P}<0.05$ represented statistical significance.

\section{Results}

\subsection{LINC01503 expression is significantly upregulated in CC cells and tissues}

We first evaluated LINC01503 expression in the GEPIA database and found that LINC01503 was higher in CC tissues relative to the normal tissues (Figure 1A). Next, we compared LINC01503 expression between $43 \mathrm{CC}$ tissues and paired healthy cervical tissues. According to the RT-qPCR assay, LINC01503 expression was higher in CC tissues in contrast with the controls (Figure 1B), and its high expression was associated with enhanced progression of CC as indicated by advanced FIGO stage, lymph-node metastasis, as well as depth of cervical invasion (Figure 1C-E). Further analysis demonstrated that LINC01503 expression in CC cell lines (SiHa, C33A, HeLa, as well as CaSki) were remarkably higher in compared to normal cervical cell line Ect1/E6E7 (Figure 1F). Intracellular localization revealed that LINC01503 was mainly expressed in the cytoplasm (Figure 1G-H). Collectively, these observations suggested that LINC01503 has a critical role in CC progression.

\subsection{LINC01503 promotes CC growth and metastasis}

To further assess the influence of LINC01503 on CC growth and metastasis, we silenced LINC01503 expressions in HeLa as well as C33A cells (Figure 2A). Results showed that LINC01503 knockdown (KD) suppressed the proliferative ability of HeLa and C33A cells relative to si-NC KD cells (Figure 2B-E). Transwell assay showed that LINC01503 inhibition suppressed cell invasion (Figure 2F).

Next, we subcutaneously xenografted stable sh-LINC01503 into nude mice and monitored tumor growth weekly. It was found that LINC01503 inhibition significantly suppressed tumor growth in vivo (Figure 3A-C). As expected, IHC analysis showed that sh-LINC01503 decreased Ki67 expressions in tumor tissues of mice (Figure 3D). Together, these data show that LINC01503 might have an oncogenic role in CC.

\subsection{LINCO1503 acts as a ceRNA by binding to miR-615-3p}

LncRNAs regulate cellular processes by functioning as ceRNA and sponging miRNAs. To explore the role of LINC01503, Starbase V2.0, LncBase v.2, Targetscan, and miRcode were used to identify the targets of LINC01503 (Figure 4A-B). The binding site for LINC01503 on miR-615-3p 3'UTR is shown in Figure 4C-D. Luciferase assay indicated that miR-615-3p mimics suppressed the fluorescence 
activity of LINC01503-WT reporter (Figure 4E). The RT-qPCR analysis showed that LINC01503 knockdown markedly enhanced miR-615-3p expression in CC cell lines (Figure 4F). RNA pull-down and RIP analysis further confirmed the relationship of LINC01503 with miR-615-3p in CC (Figure 4G-H).

We further assessed miR-615-3p expression in CC by RT-qPCR and observed that the levels of miR-615-3p were remarkably lower in CC tissues and cell lines (Figure 5A-B). Correlation analysis indicated a crosstalk between LINC01503 and miR-615-3p in CC tissues (Figure 5C). Evaluation of the cellular functions of miR-615-3p using Edu and transwell invasion assays showed that the proliferative as well as the invasion ability of HeLa cells were inhibited when miR-615-3p was upregulated (Figure 5D-E). Together, these data indicate that LINC01503 might

A

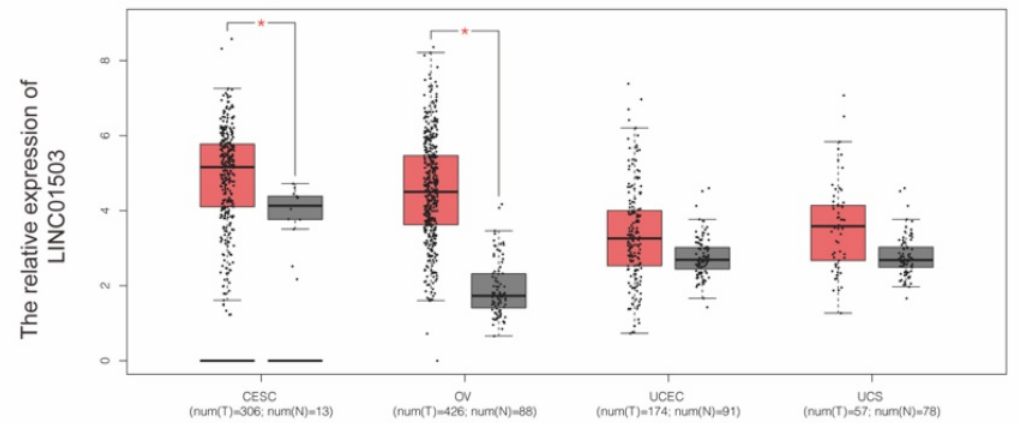

C

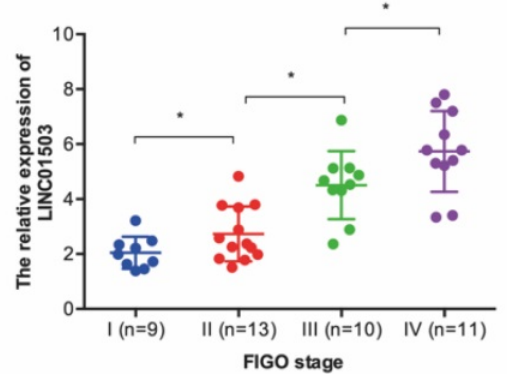

$\mathrm{F}$
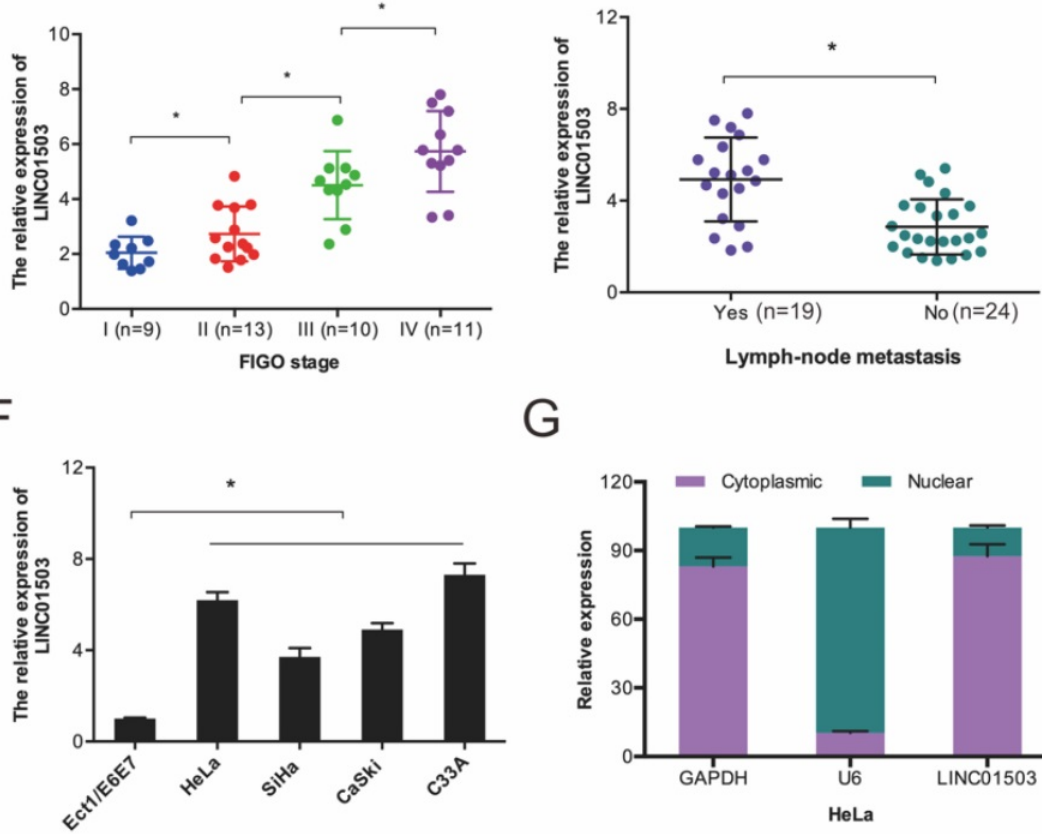

G

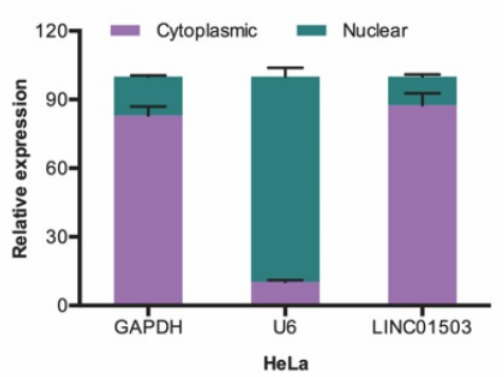

regulate the progression of $\mathrm{CC}$ by interacting with miR-615-3p.

\subsection{LINC01503 positively modulates CCNDI by sponging miR-615-3p}

To investigate the mechanism of miR-615-3p in CC cells, we conducted target prediction analysis on TargetScan, miRTarBase, and miRWalk databases and identified CCND1 as a promising miR-615-3p target (Fig. 6A-B). Dual-luciferase reporter assessment found that miR-615-3p mimics remarkably repressed luciferase enzyme activity in CCND1-WT (Figure 6C). Next, we assessed the influence of miR-615-3p on CCDN1 expression. Western blot assay demonstrated that miR-615-3p overexpression reduced the expression levels of CCND1 in HeLa and C33A cells (Figure 6D-E).

B

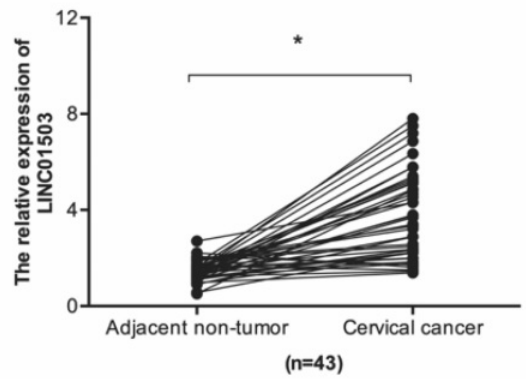

E

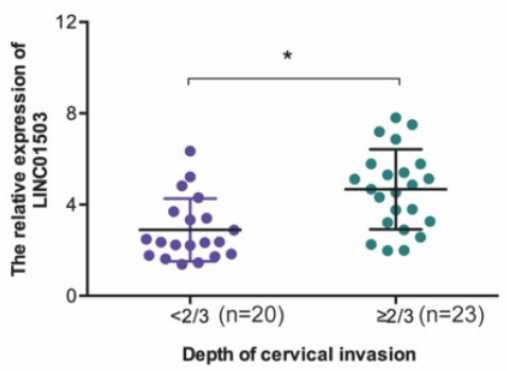

$\mathrm{H}$

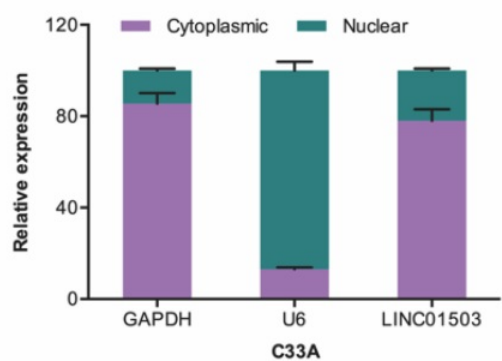

Figure 1: LINC01503 expression is enhanced in CC. (A) LINC01503 expression LINC01503, as determined on the GEPIA database. (B) RT-qPCR analysis of LINC01503 expression in 43 paired CC tissues. (C-E) High levels of LINC01503 positively linked to advanced FIGO stage, lymph node metastasis, as well as depth of cervical invasion in patients. (F) RT-qPCR analysis of LINC01503 expression in CC cells. (G, H) The subcellular localization of LINC01503 as analyzed by subcellular fractionation assay. OV: Ovarian serous cystadenocarcinoma; CESE: Cervical squamous cell carcinoma and endocervical adenocarcinoma; UCEC: Uterine Corpus Endometrial Carcinoma; UCS: Uterine Carcinosarcoma. $* P \leq 0.05$. 
A

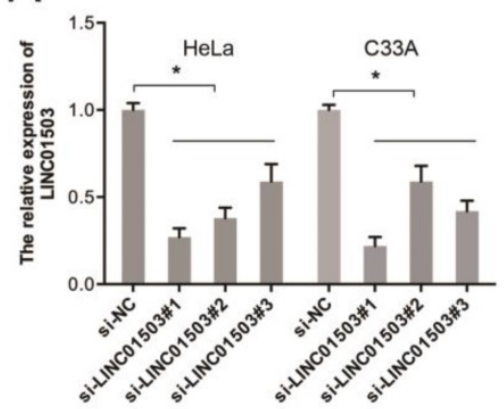

B

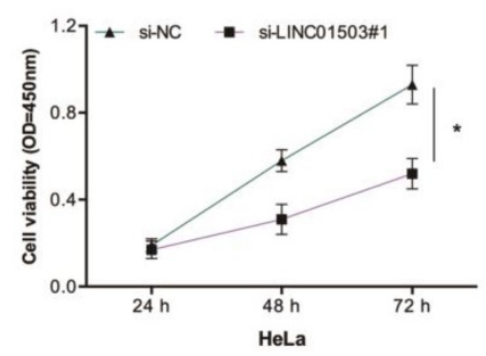

D

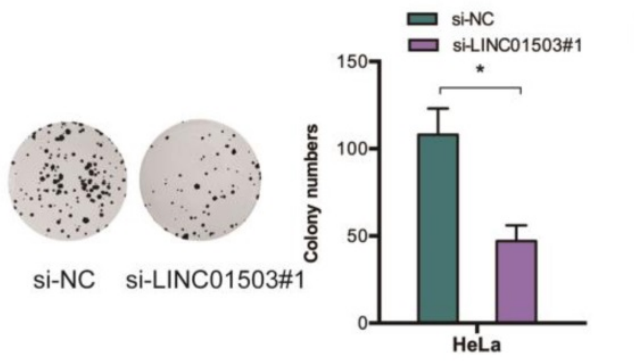

C

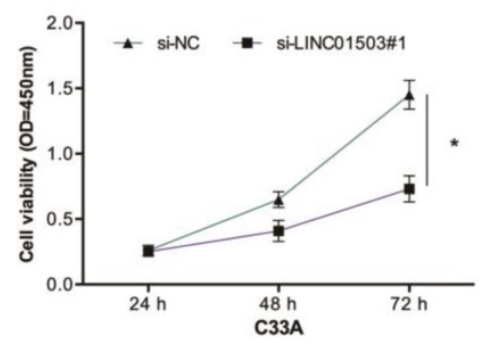

E

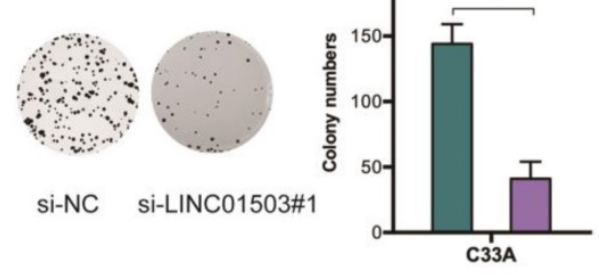

F
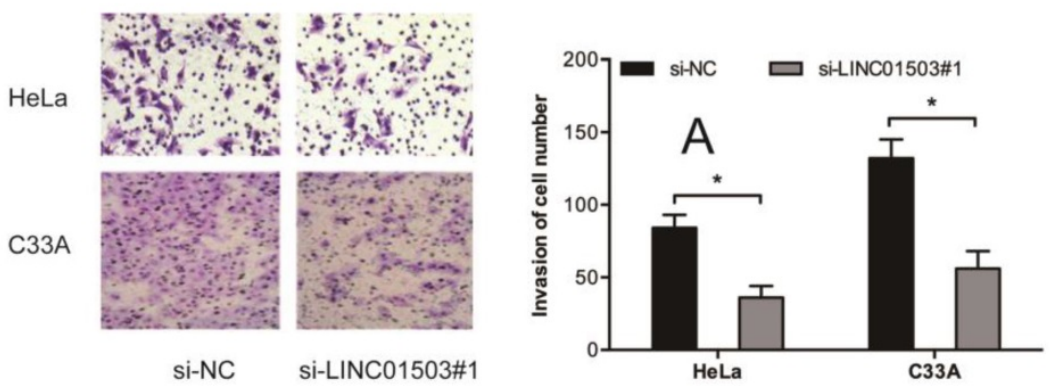

Figure 2: LINC01503 enhances the invasion and proliferative ability of CC cells. (A) HeLa, C33A cells were infected with si-NC or si-LINC01503 vectors. (B-E) LINC01503 inhibition suppresses the viability of CC cells as demonstrated by CCK-8 as well as colony formation assays. (F) LINC01503 KD decreases CC cell invasion. $* P \leq 0.05$.

A
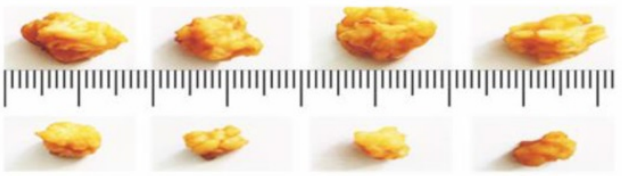

sh-LINC01503

B

sh-NC

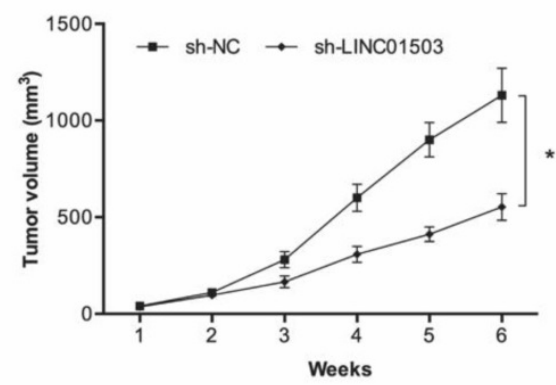

C

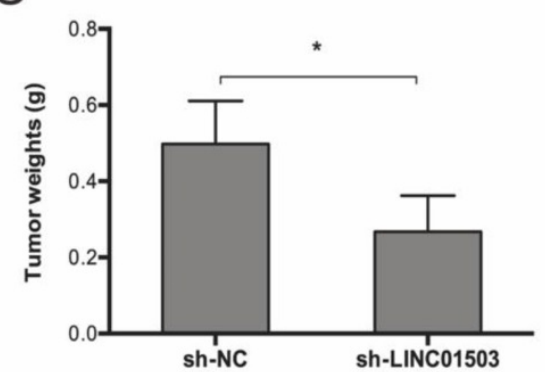

$\mathrm{D}$
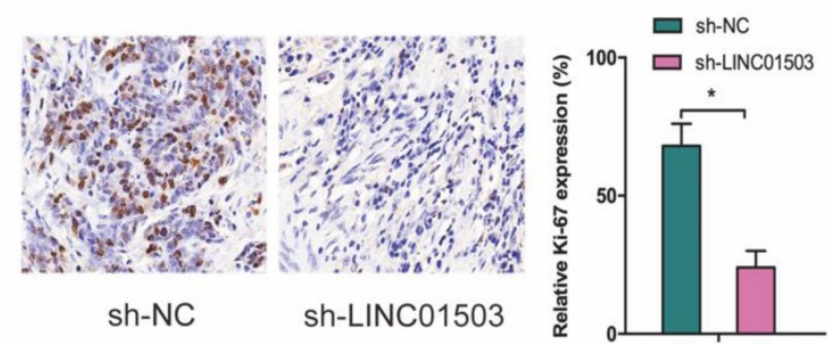

Figure 3: LINC01503 promotes CC growth in vivo. (A-C) LINC01503 suppression reduces tumor volume and weight. (D) Assessment of Ki67 expression in tumor tissue by IHC assay. $* P \leq 0.05$ 
A

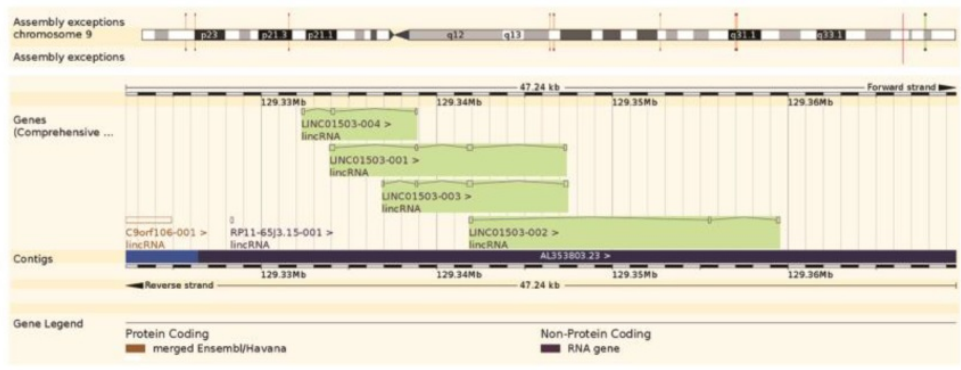

C

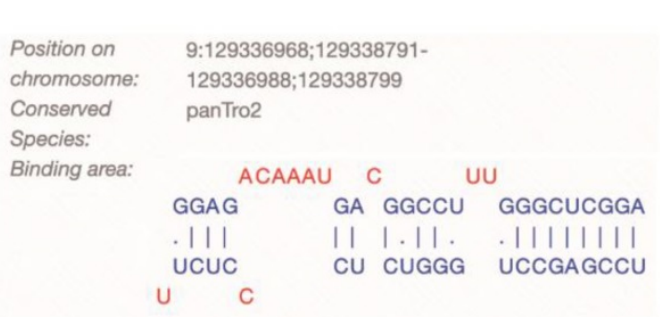

F
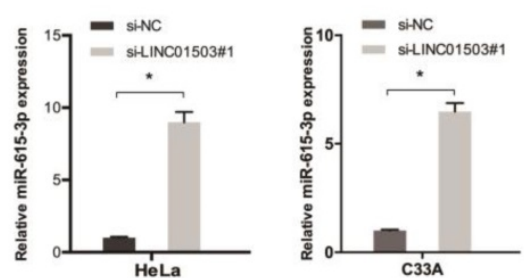

D

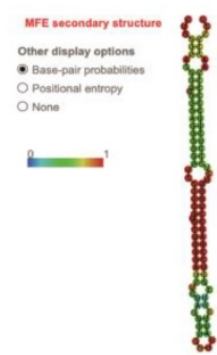

B

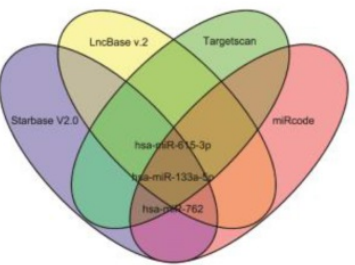

E

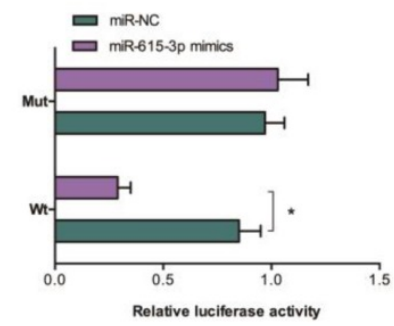

$\mathrm{H}$

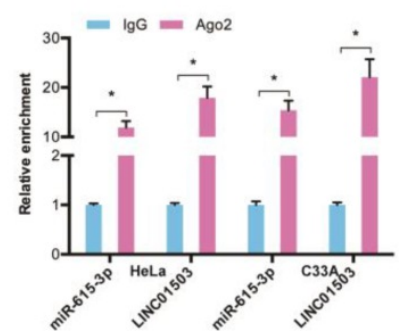

Figure 4: LINC01503 acts as ceRNA by binding to miR-615-3p. (A) Information of LINC01503. (B) Venn diagram showing the potential targets of LINC01503. (C) The biding site of LINC01503 on miR-615-3p. (D) The secondary structure of miR-615-3p. (E) miR-615-3p mimic reduces the fluorescence activity of LINC01503-WT group. (F) Silencing of LINC01503 significantly increased miR-615-3p expression (G, H) RNA pull-down and RIP analysis validated the association of LINC01503 with miR-615-3p in CC. *P $\leq 0.05$.

A

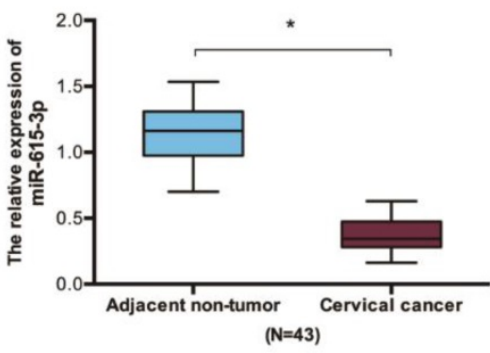

D

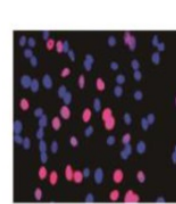

miR-NC

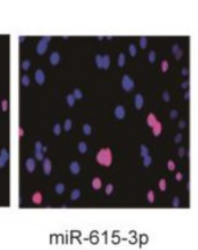

B

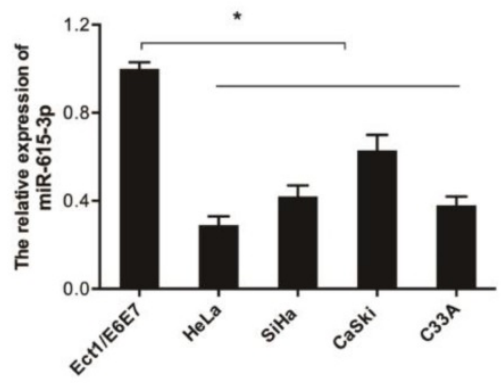

$\mathrm{E}$
C

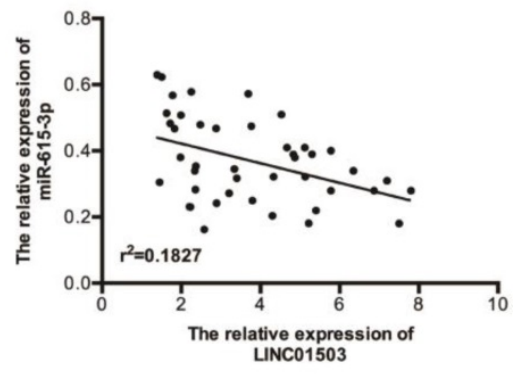

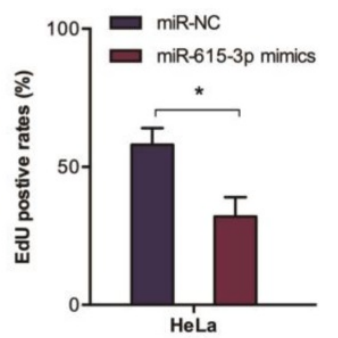
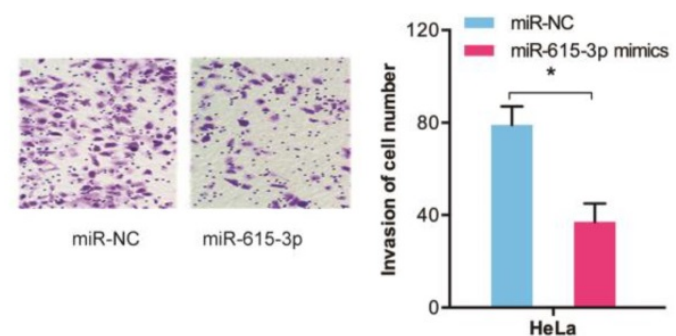

Figure 5: The function of miR-615-3p in CC. (A, B) RT-qPCR determination of miR-615-3p expression in CC tissues as well as cells. (C) Crosstalk between LINC01503 expression and miR-615-3p in CC tissues. (D, E) Edu and transwell invasion assays verified that miR-615-3p overexpression reduces $C C$ cell viability and invasion. $* P \leq 0.05$. 
A

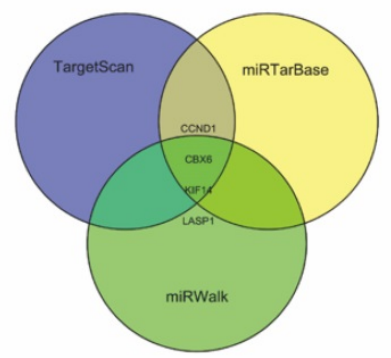

C

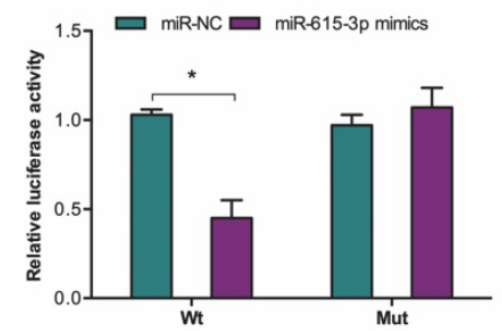

F

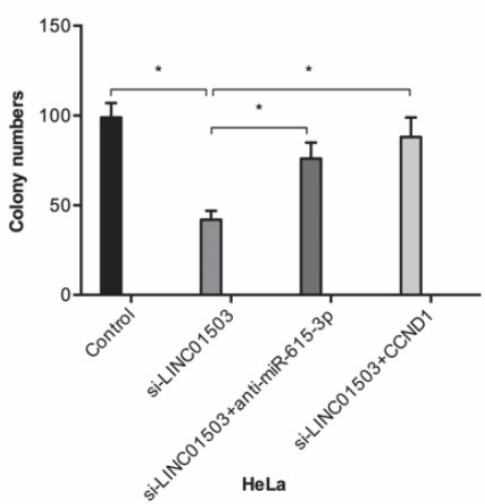

B

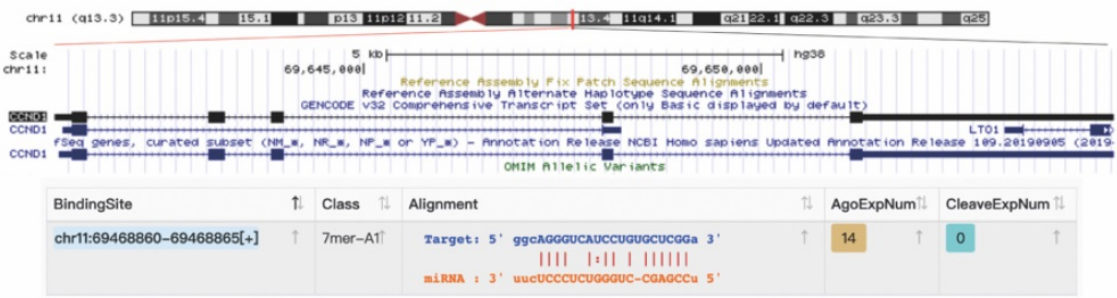

E
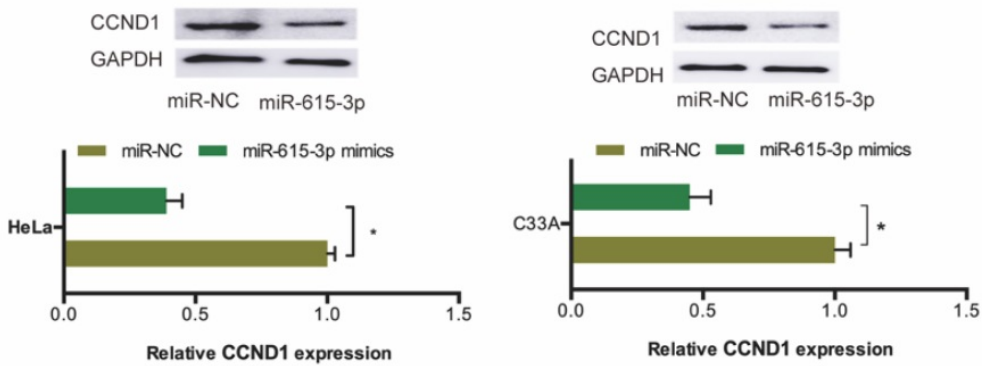

G

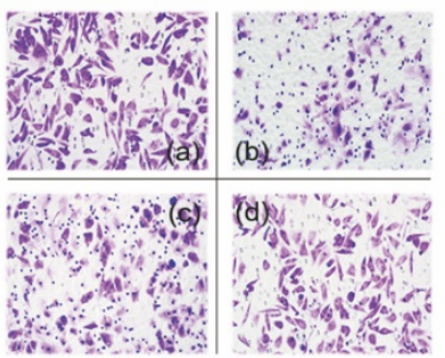

$\begin{array}{ll}\text { (a) Control } & \text { (b) si-LINC01503 }\end{array}$

(c) si-LINC01503+anti-miR-615-3p

(d) si-LINC01503+CCND1

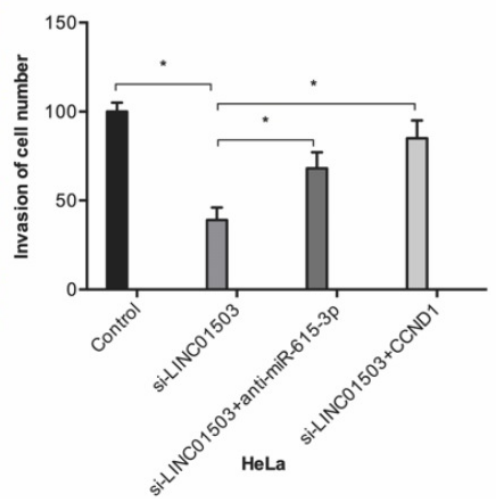

Figure 6: miR-615-3p targets CCNDI in CC. (A) Venn diagrams showing the potential miR-615-3p targets. (B) The biding site of CCNDI on miR-615-3p. (C) Relative luciferase enzyme activity was assessed in CC cells co-transfected with CCNDI-WT, CCNDI-MUT, miR-NC, or miR-615-3p mimic. (D, E) miR-615-3p overexpression reduces CCNDI protein levels in CC cells. $(\mathrm{F}, \mathrm{G})$ miR-615-3p inhibitors or CCNDI overexpression abolished the regulation of si-LINC01503 on $C C$ cell proliferation along with invasion. $* P \leq 0.05$.

Furthermore, we performed rescue assays to verify the involvement of LINC01503/miR-615-3p/ CCND1 axis in CC progression. Colony formation and transwell invasion assays revealed that miR-615-3p inhibitors or CCND1 upregulation significantly obliterated the suppression of Hela cell proliferation as well as invasion by si-LINC01503 (Figure 6F-G). This indicating that LINC01503 promotes the progression of $\mathrm{CC}$ cells by interacting with the miR-615-3p/CCND1 axis.

\section{Discussion}

Accumulating evidence indicates that lncRNAs influences the progression of diseases [13, 14]. The recently discovered lncRNA, LINC01503 is located on chromosome 9:129,336,879-129,347,464 in humans. It has been reported that LINC01503 influences tumorigenesis. For instance, Xie et al. reported that LINC01503 is highly expressed in squamous cell carcinoma and promotes proliferation, migration, invasion, as well as the growth of tumor xenografts [15]. Ding et al. showed that LINC01503 enhances the invasion and proliferation of tumor cells by regulating Wnt signaling in gastric cancer [16]. Lu et al. found that LINC01503 facilitates progression of colorectal cancer via the miR-4492/FOXK1 axis [17]. Moreover, Peng et al. found that LINC01503 enhances the progression of tumor cells by modulating miR-342-3p /FXYD3 axis in CC [18]. However, the roles of LINC01503 in CC development and progression remains unclear.

Here, we established that LINC01503 was upregulated in CC cells as well as tissues and that this associated with advanced clinical characteristics. 
Additionally, we observed that LINC01503 knockdown inhibited CC progression by suppressing the metastatic and proliferative abilities of tumor cells, but the precise mechanism is yet to be revealed.

LncRNAs have been found to influence tumorigenesis by sponging miRNAs [19]. Several studies have found that lncRNA functions as ceRNAs to sponge miRNAs in CC. Zhu et al. revealed that TUG1 sponges miR-138-5p to promote CC progression [20]. Yang et al. indicated that lncRNA OIP5-AS1 enhances the invasion and proliferative abilities of CC cells by upregulating ITGA6 and sponging miR-143-3p [21]. Here, we report that LINC01503 might function as a ceRNA for miR-615-3p in CC. LINC01503 suppression upregulated miR-615-3p in CC cells. Furthermore, using luciferase reporter, RNA pull-down, as well as RIP assays, we verified the cross talk between LINC01503 and miR-615-3p. Moreover, miR-615-3p was demonstrated to be poorly expressed in CC tissues and it suppressed tumor growth along with invasion in vitro. Collectively, our data demonstrates that miR-615-3p mediates the oncogenic function of LINC01503 in CC.

CCND1, along with cyclin-dependent kinases, modulates G1/S transition through $\mathrm{Rb}$ phosphorylation [22, 23]. Accumulating evidence shows that CCND1 influences a plethora of biological processes associated with the pathogenesis, as well as drug resistance in human cancers [24, 25]. CCND1 is therefore recognized as an oncogene in multiple cancer types consisting of lung cancer, osteosarcoma, and nasopharyngeal carcinoma et al [24-26]. Nevertheless, the function of CCND1 in CC is unknown. Here, we found that CCND1 is a target of miR-615-3p in CC and is negatively modulated by miR-615-3p. We showed that higher levels of CCND1 abolish the suppressive effects of LINC01503 KD on CC cell proliferation and invasion. Collectively, the results indicate that LINC01503 knockdown suppresses CC progression by interacting with the miR-615-3p/CCND1 axis.

In conclusion, our data show that LINC01503 is upregulated in CC and its knockdown suppresses the invasion and proliferative ability of CC cells. Therefore, it modulates miR-615-3p expression, thereby suppresses CCND1 expression. Our study highlights the therapeutic potential of targeting the LINC01503/miR-615-3p/CCND1 axis in the treatment of CC.

\section{Abbreviations}

CC: Cervical cancer; CESE: Cervical squamous cell carcinoma and endocervical adenocarcinoma; $\mathrm{OV}$ : Ovarian serous cystadenocarcinoma; UCEC: Uterine
Corpus Endometrial Carcinoma; UCS: Uterine Carcinosarcoma; lncRNAs: Long non-coding RNAs; miRNAs: MicroRNAs; Mut: Mutant; WT: Wild type; UTR: Untranslated region.

\section{Acknowledgments}

\section{Availability of data and materials}

The datasets supporting the conclusions of this article are included within the article.

\section{Ethics approval and consent to participate}

This research was approved by Cangzhou Central Hospital and conducted in accordance with the principles of the Declaration of Helsinki.

\section{Author Contributions}

JF, FYG, JX conceived and designed this study. JF, YYL, XYX conducted the experiments and analyzed and checked the data. JF, JX supervised the whole project and revised the manuscript. All authors read and approved the final manuscript.

\section{Competing Interests}

This article has not been published elsewhere in whole or in part. All authors have read and approved the content and agree to submit for consideration for publication in the journal. The authors declared no conflict of interest.

\section{References}

1. Bray F, Ferlay J, Soerjomataram I, et al. Global cancer statistics 2018: GLOBOCAN estimates of incidence and mortality worldwide for 36 cancers in 185 countries[J]. CA: a cancer journal for clinicians, 2018, 68(6): 394-424.

2. Franco E L, Duarte-Franco E, Ferenczy A. Cervical cancer: epidemiology, prevention and the role of human papillomavirus infection[J]. Cmaj, 2001, 164(7): 1017-1025.

3. Shi J F, Canfell K, Lew J B, et al. The burden of cervical cancer in China: synthesis of the evidence[J]. International journal of cancer, 2012, 130(3): 641-652

4. Arbyn M, Castellsagué X, de Sanjosé S, et al. Worldwide burden of cervical cancer in 2008[J]. Annals of oncology, 2011, 22(12): 2675-2686.

5. Mercer T R, Dinger M E, Mattick J S. Long non-coding RNAs: insights into functions[J]. Nature reviews genetics, 2009, 10(3): 155-159.

6. Fatica A, Bozzoni I. Long non-coding RNAs: new players in cell differentiation and development[J]. Nature Reviews Genetics, 2014, 15(1): 7-21.

7. $\mathrm{Li} \mathrm{C} \mathrm{H}$, Chen Y. Targeting long non-coding RNAs in cancers: progress and prospects[J]. The international journal of biochemistry \& cell biology, 2013, 45(8): 1895-1910.

8. He R Z, Luo D X, Mo Y Y. Emerging roles of lncRNAs in the post-transcriptional regulation in cancer[J]. Genes \& diseases, 2019, 6(1): 6 .

9. Tang Y, Wang J, Lian Y, et al. Linking long non-coding RNAs and SWI/SNF complexes to chromatin remodeling in cancer[J]. Molecular cancer, 2017, 16(1): 42.

10. Huang $\mathrm{M}$, Wang $\mathrm{H}, \mathrm{Hu} \mathrm{X}$, et al. IncRNA MALAT1 binds chromatin remodeling subunit BRG1 to epigenetically promote inflammation-related hepatocellular carcinoma progression[J]. Oncoimmunology, 2019, 8(1): e1518628.

11. Xiu D, Liu L, Cheng M, et al. Knockdown of lncRNA TUG1 Enhances Radiosensitivity of Prostate Cancer via the TUG1/miR-139-5p/SMC1A Axis[J]. OncoTargets and therapy, 2020, 13: 2319.

12. Hu Y H, Sun J, Zhang J, et al. Long non-coding RNA ROR sponges miR-138 to aggravate hypoxia/reoxygenation-induced cardiomyocyte apoptosis via upregulating Mst1[J]. Experimental and Molecular Pathology, 2020: 104430.

13. Peng L, Yuan X, Jiang B, et al. LncRNAs: key players and novel insights into cervical cancer[J]. Tumor Biology, 2016, 37(3): 2779-2788.

14. Peng W X, Koirala P, Mo Y Y. LncRNA-mediated regulation of cell signaling in cancer[J]. Oncogene, 2017, 36(41): 5661-5667. 
15. Xie J J, Jiang Y Y, Jiang $Y$, et al. Super-enhancer-driven long non-coding RNA LINC01503, regulated by TP63, is over-expressed and oncogenic in squamous cell carcinoma[J]. Gastroenterology, 2018, 154(8): 2137-2151. e1.

16. Ding J, Shi F, Xie G, et al. Long Non-coding RNA LINC01503 Promotes Gastric Cancer Cell Proliferation and Invasion by Regulating Wnt Signaling[J]. Digestive Diseases and Sciences, 2020: 1-8.

17. Lu S R, Li Q, Lu J L, et al. Long non-coding RNA LINC01503 promotes colorectal cancer cell proliferation and invasion by regulating miR-4492/FOXK1 signaling[J]. Experimental and therapeutic medicine, 2018, 16(6): 4879-4885

18. Peng X, Gao J, Cai C, et al. LncRNA LINC01503 aggravates the progression of cervical cancer through sponging miR-342-3p to mediate FXYD3 expression[J]. Bioscience Reports, 2020, 40(6): BSR20193371.

19. Zhu J, Yu W, Wang Y, et al. lncRNAs: function and mechanism in cartilage development, degeneration, and regeneration[J]. Stem cell research \& therapy, 2019, 10(1): 1-12.

20. Zhu J, Shi $\mathrm{H}$, Liu $\mathrm{H}$, et al. Long non-coding RNA TUG1 promotes cervical cancer progression by regulating the miR-138-5p-SIRT1 axis[J]. Oncotarget, 2017, 8(39): 65253

21. Yang J, Jiang B, Hai J, et al. Long noncoding RNA opa-interacting protein 5 antisense transcript 1 promotes proliferation and invasion through elevating integrin a6 expression by sponging miR-143-3p in cervical cancer[J]. Journal of cellular biochemistry, 2019, 120(1): 907-916.

22. Tetsu O, McCormick F. $\beta$-Catenin regulates expression of cyclin D1 in colon carcinoma cells[J]. Nature, 1999, 398(6726): 422-426.

23. Qie S, Diehl J A. Cyclin D1, cancer progression, and opportunities in cancer treatment[J]. Journal of molecular medicine, 2016, 94(12): 1313-1326.

24. Fang $C$, Jiang B, Shi $X$, et al. Hes3 enhances the malignant phenotype of lung cancer through upregulating Cyclin D1, Cyclin D3 and MMP7 expression[J]. International journal of medical sciences, 2019, 16(3): 470.

25. Yu X, Pang L, Yang T, et al. IncRNA LINC01296 regulates the proliferation, metastasis and cell cycle of osteosarcoma through cyclin D1[J]. Oncology reports, 2018, 40(5): 2507-2514.

26. Nurhidayat A A, Afiati $\mathrm{H}$ A U, Hernowo B S. The role of cyclin D1 and vascular endothelial growth factor (VEGF) in radiotherapy response of undifferentiated nasopharyngeal carcinoma[J]. J Med Sci, 2018, 50(4): 385-391. 